\title{
Autologous Bone Augmentation in Combination with an Ameloblastoma in the Maxillary Region- A Case Report?
}

\author{
Alevizakos Vasilios ${ }^{1}$, Stoetzer Marcus ${ }^{2}$ and von See Constantin ${ }^{2}$ \\ ${ }^{1}$ Danube Privat University, Steiner Landstraße 124, 3500 Krems an der Donau, Austria \\ ${ }^{2}$ Medical High School Hannover, Department of oral and maxillofacila surgery, Carl-Neuberg-Straße 1, 30657 \\ Hannover, Germany \\ "Corresponding Authors: Dr. Marcus Stoetzer, Medical High School Hannover, Department of Oral and \\ Maxillofacial Surgery, Carl-Neuberg-Straße 1, 30657 Hannover, Germany, Tel: +495115324747; E-mail: \\ marcus_stoetzer@web.de
}

Received: 02 February 2018; Accepted: 02 March 2018; Published: 08 March 2018

\begin{abstract}
Maxillary ameloblastomas are rare odontogenic tumours. This case report describes a patient who presented with an ameloblastoma in the maxillary molar region. In this case, bone surrounding tooth 28 was removed together with the tooth itself and the cystic mass to perform an augmentation at 11 for subsequent implant placement. Histological examination of the mass revealed it to be an ameloblastoma. This called for an extensive follow-up resection at tooth 28 and removal of the augmentation material at 11 .
\end{abstract}

Keywords: Odontogenic tumour; Bone augmentation; Maxilla

\section{Introduction}

Odontogenic tumours are neoplasms of tissues involved in odontogenesis. An ameloblastoma usually develops from the odontogenic epithelium during formation of the dental lamina [1, 2]. It is locally aggressive and destructive, but metastasises only rarely [3, 4]. Ameloblastomas mostly affect patients in their third and fourth decade of life [2]. Treatment of radicular cysts consists of cystectomy with subsequent spontaneous bone healing. The use of bone substitute material is somewhat controversial and depends on the augmentation volume $[5,6]$. 


\section{Case Report}

A 57-year-old patient with no systemic diseases presented with complaints about his much-elongated upper central incisor tooth (11), insufficient crowns and fillings as well as missing teeth. He was dissatisfied with his aesthetic appearance.

\subsection{Radiological examination}

A panoramic dental X-ray was taken (Figure 1). Apical to tooth 28 , a clearly defined ellipsoid translucency beginning at the maxillary tuberosity, touching the floor of the maxillary sinus and the alveolar crest was further diagnosed. We suspected an odontogenic cyst, particularly a radicular cyst.

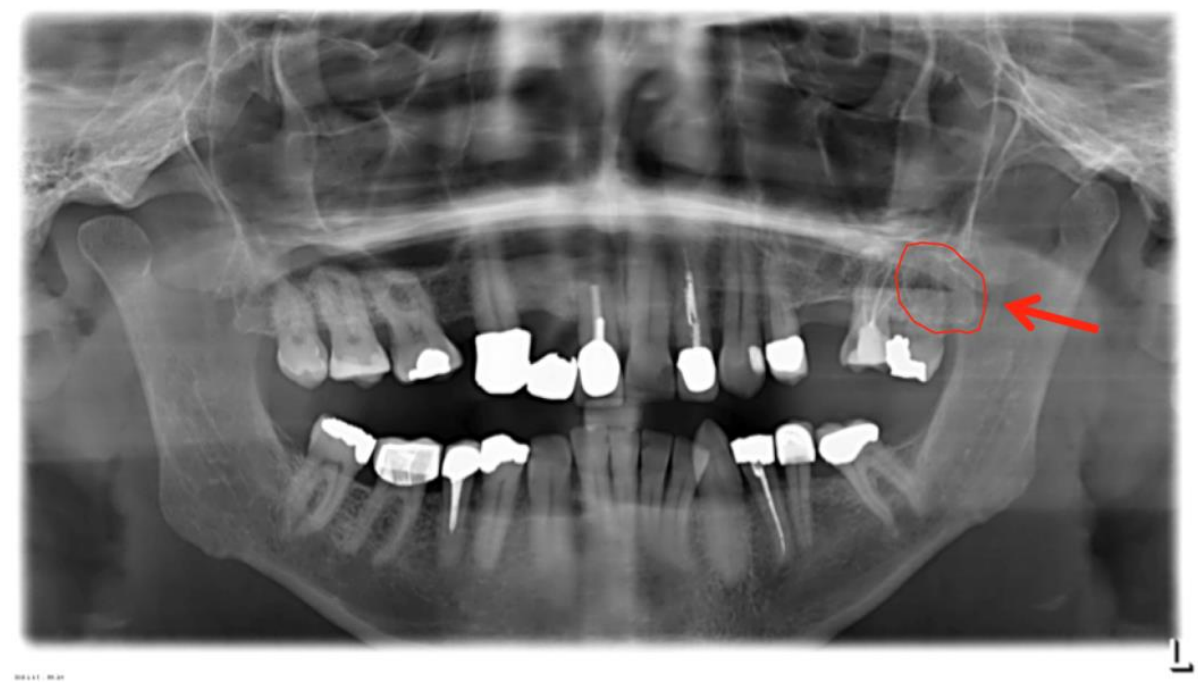

Figure 1: Panoramic X-ray after initial examination.

\subsection{Treatment and diagnosis}

After preoperative planning based on situation models and radiological and clinical evaluation of the oral cavity, it was decided to remove the tooth 11, 12 and 28. Preoperative implant planning showed that the region of the teeth to be extracted needed buccal augmentation. Extraction of tooth 11, osteotomy of tooth 28 and bone augmentation with bonechips at 11 were performed. The entirety of the removed tissue periapical to tooth 28 was suspected to be a radicular cyst and was forwarded for histological examination. The wound was closed with local flap surgery (vicryl 3-0 suture).

\subsection{Histological examination and diagnosis}

Histological sections described material revealed a cystic lesion with collagenous connective tissue at the outside. Tumour cells were of medium size, moderately to slightly pleomorphic with a shift in the nuclear-to-cytoplasmic ratio in favour of cell nuclei. They were elements of an ameloblastoma of a mostly plexiform, focal as well as follicular type and with the clinical picture of a cystic lesion (Figure 2). 


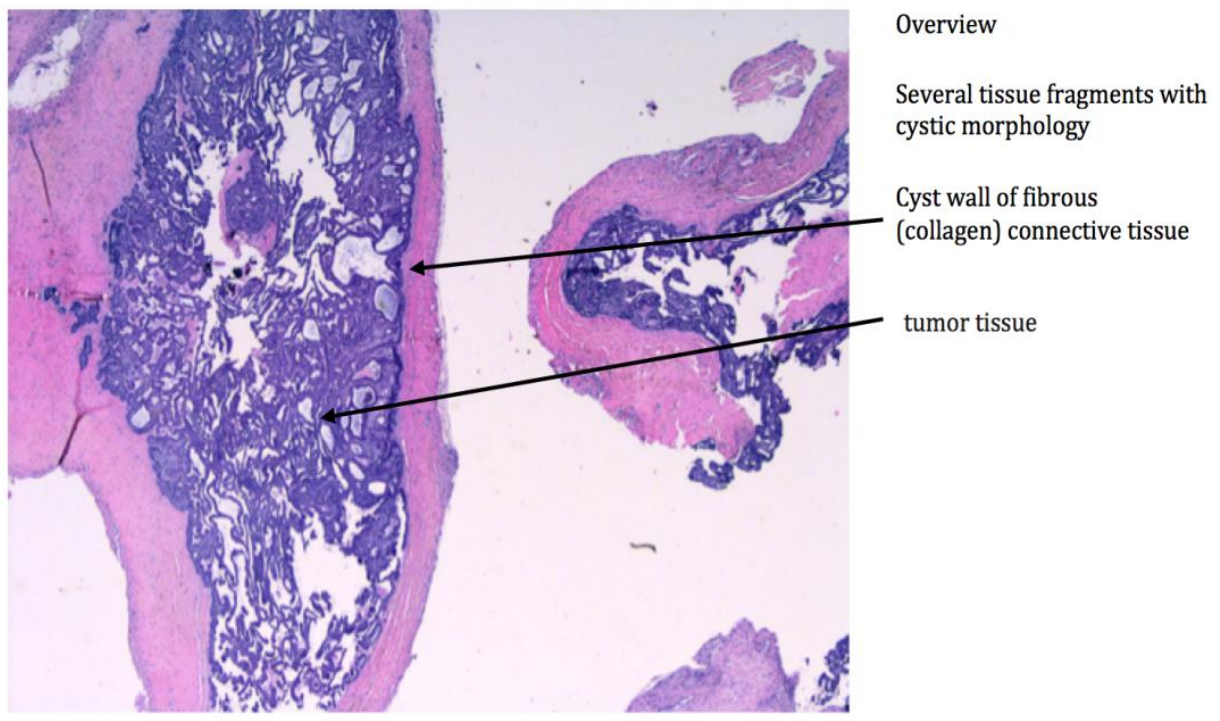

Figure 2: Histological examination.

\subsection{Postoperative treatment}

From the histological diagnosis followed the indication to remove the augmentation material in the region of the front teeth and to perform a follow-up resection in region 28. After a four-month healing period, treatment at the surgical site was continued. Further implantological treatment was eschewed in favour of a conventional metalveneer ceramic bridge restoration from tooth 16 to 22 (Figure 3).

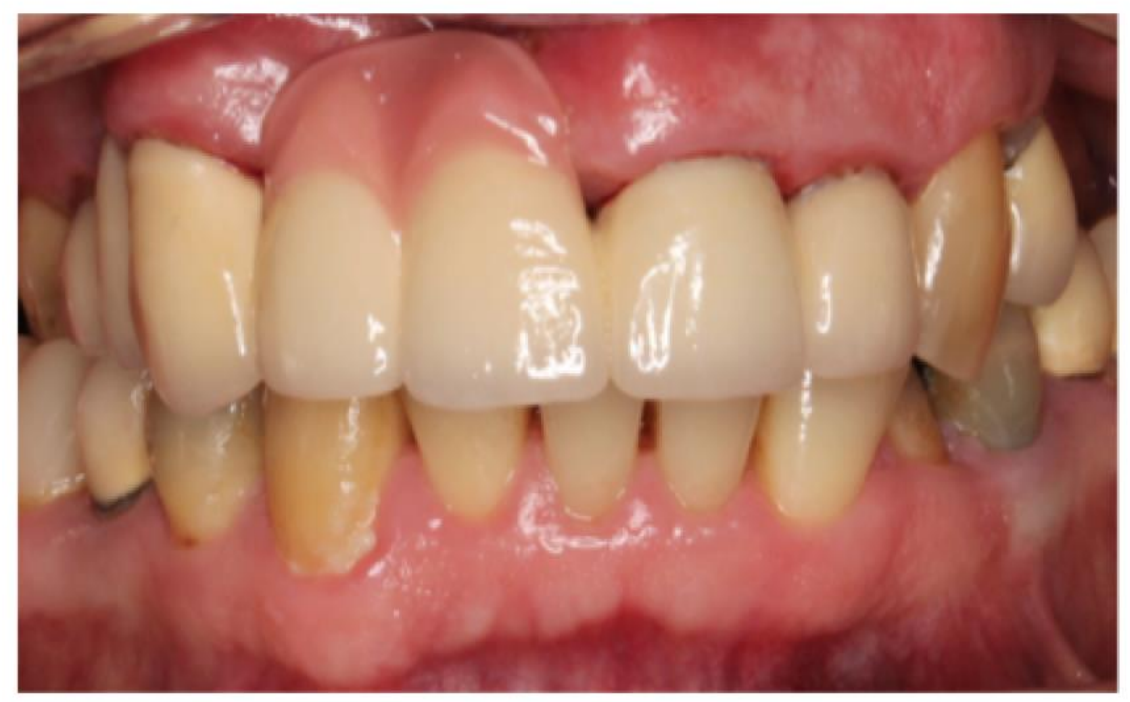

Figure 3: Prosthetic outcome. 


\section{Discussion}

Ameloblastomas, belong to the group of odontogenic tumours [7]. In the literature, a unilocular translucency found on X-ray with a clear demarcation and in local relation to an insufficiently restored tooth is almost invariably identified as inflammatory cyst upon histological examination [8]. Histological diagnosis of tissue specimens is thus not regularly performed in such case. With increasing growth, it causes tooth displacement and root resorption. According to the current literature, this type of tumour is very rare in this decade of life and in this location, and the majority of these translucencies are histologically diagnosed as odontogenic cyst [9]. In order to resect potential daughter cysts, ameloblastoma resections require a safe margin of 1-2 cm, which here clearly exceeded the harvesting region [10].

\section{Conclusion}

Radiological and clinical examinations often allow for a tentative diagnosis that is based on available literature and epidemiology that provide information on the incidence and prevalence of known diseases. The case presented in this paper serves to demonstrate that histological examination of tissue specimens prior to tissue grafting may be advisable in order to avoid inadvertent introduction of pathogenic cells into healthy tissue.

\section{Acknowledgement}

Our special thanks go to the Department of Pathology of the University Hospital of Krems for the histological pictures and to the Department of Oral and Maxillofacial Surgery of the University Hospital of Sankt Pölten for further treatment.

\section{References}

1. Fernandes AM, Duarte EC, Pimenta FJ, et al. Odontogenic tumors: a study of 340 cases in a Brazilian population. J Oral Pathol Med 34 (2005): 583-587.

2. Iordanidis S, Makos C, Dimitrakopoulos J, et al. Ameloblastoma of the maxilla. Case report. Aust Dent J 44 (1999): 51-55.

3. Ikemura K, Tashiro H, Fujino H, et al. Ameloblastoma of the mandible with metastasis to the lungs and lymph nodes. Cancer 29 (1972): 930-940.

4. Barrett AW, Sneddon KJ, Tighe JV, et al., Dentigerous Cyst and Ameloblastoma of the Jaws. Int J Surg Pathol 5 (2017): 141-147.

5. Chacko R, Kumar S, Paul A, et al. Spontaneous Bone Regeneration After Enucleation of Large Jaw Cysts: A Digital Radiographic Analysis of 44 Consecutive Cases. J Clin Diagn Res 9 (2015): Zc84-89.

6. Ihan Hren N, Miljavec M. Spontaneous bone healing of the large bone defects in the mandible. Int J Oral Maxillofac Surg 37 (2008): 1111-1116.

7. Ali K, Munir F, Rehman A, et al. Clinico-radiographic study of odontogenic cysts at a tertiary care centre. J Ayub Med Coll Abbottabad 26 (2014): 92-94.

8. Chaine A, Pitak-Arnnop P, Dhanuthai K, et al. An asymptomatic radiolucent lesion of the maxilla. Oral Surgery, Oral Medicine, Oral Pathology, Oral Radiology and Endodontology 107 (2009): 452-457. 
9. Gunawardhana KS, Jayasooriya PR, Tilakaratne WM. Diagnostic dilemma of unicystic ameloblastoma: novel parameters to differentiate unicystic ameloblastoma from common odontogenic cysts. J Investig Clin Dent 5 (2014): 220-225.

10. Smullin SE. Oral Surg Oral Med oral Pathol oral Radiol Endod 2008; 105: 37-40; Paikkatt VJ et al. Indian Soc Pedoded Prev Dent 25 (2007): 106-110.

Citation: Alevizakos Vasilios, Stoetzer Marcus, von See Constantin. Autologous Bone Augmentation in Combination with an Ameloblastoma in the Maxillary Region- A Case Report?. Archives of Clinical and Medical Case Reports 2 (2018): 45-49.

(C) 1 (P) $\begin{aligned} & \text { This article is an open access article distributed under the terms and conditions of the } \\ & \text { Creative Commons Attribution (CC-BY) license 4.0 }\end{aligned}$ 